\title{
Closed-Loop Control in Fused MR-TRUS Image-Guided Prostate Biopsy
}

\author{
Sheng $\mathrm{Xu}^{1}$, Jochen Kruecker ${ }^{1}$, Peter Guion ${ }^{2}$, Neil Glossop ${ }^{3}$, Ziv Neeman ${ }^{2}$, \\ Peter Choyke ${ }^{2}$, Anurag K. Singh ${ }^{2}$, and Bradford J. Wood ${ }^{2}$ \\ ${ }^{1}$ Philips Research North America, Briarcliff, NY 10510, USA \\ \{sheng.xu, jochen. kruecker\} @philips.com \\ ${ }^{2}$ National Institutes of Health, Bethesda, MD 20892, USA \\ ${ }^{3}$ Traxtal Inc, Toronto, ON M5V 2J1, Canada
}

\begin{abstract}
Multi-modality fusion imaging for targeted prostate biopsy is difficult because of prostate motion during the biopsy procedure. A closed-loop control mechanism is proposed to improve the efficacy and safety of the biopsy procedure, which uses real-time ultrasound and spatial tracking as feedback to adjust the registration between a preoperative 3D image (e.g. MRI) and realtime ultrasound images. The spatial tracking data is used to initialize the imagebased registration between intraoperative ultrasound images and a preoperative ultrasound volume. The preoperative ultrasound volume is obtained using a $2 \mathrm{D}$ sweep and manually registered to the MRI dataset before the biopsy procedure. The accuracy of the system is $2.3 \pm 0.9 \mathrm{~mm}$ in phantom studies. The results of twelve patient studies show that prostate motion can be effectively compensated using closed-loop control.
\end{abstract}

Keywords: motion compensation, prostate biopsy, image registration.

\section{Introduction}

Prostate cancer is the most common non-skin cancer and the second leading cause of cancer death among American men [1]. Transrectal ultrasound (TRUS) guided needle biopsy is the most frequently used method for diagnosing prostate cancer due to its real-time nature, low cost, and simplicity [2]. However, the use of ultrasound (US) to detect prostate cancer is limited by its relatively poor image quality and low sensitivity and specificity for prostate cancers. It is difficult to use US for targeted biopsy guidance because most cancers are not visible sonographically. Magnetic resonance imaging (MRI) is superior for visualizing the prostate anatomy and focal lesions suspicious for prostate cancer. However, MRI imaging is costly and the magnetic environment makes interventional procedures more complex thus making MRI imaging unsuitable as an intra-procedural modality for routine biopsy guidance.

Since preoperative MRI and real-time US complement each other, it is desirable to fuse them and take advantage of the superior visualization of MRI images in TRUS guided biopsy [3]. Several systems have been presented in literature for image fusion 
of preoperative MRI (or CT) images and real-time US images [4][5]. In these systems, the ultrasound probe is tracked by a localizer that assigns a global coordinate system to the US images. The registration between the MRI image and the localizer is obtained using fiducial markers before the surgical intervention. After both MRI and US are registered to the localizer, multi-planar reconstruction of the MRI image can be computed and overlaid on the 2D US image in real-time.

It should be noted that these systems only work well if the target is static relative to the fiducial markers. Unfortunately, the prostate moves considerably in the pelvic cavity for several reasons: First, the patient often moves involuntarily due to pain or pressure related to the needle insertion; Second, the transrectal ultrasound probe itself can move and distort the prostate. Finally, respiratory motion of the patient may shift the prostate [6]. It is apparent that skin fiducials are not very useful for the motion correction. In our earlier work [7], gold seeds were implanted into the prostate. This approach was abandoned because very few seeds could be identified in both MRI and US. Without intraoperative feedback to account for prostate motion, the system features an open-loop control mechanism. Since the prostate is a very small organ, the motion can easily result in loss of accuracy in the MRI/US fusion display, leading to inaccurate needle insertions when using the fused display for targeted biopsies. Sometimes, MRI and US images can be completely disconnected from each other, making the MRI image useless for surgical navigation.

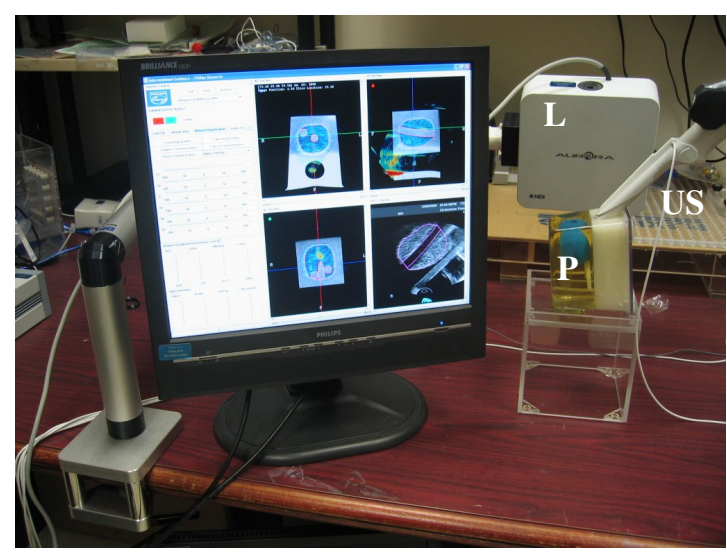

Fig. 1. System components: localizer (L), tracked ultrasound probe (US) and prostate phantom (P)

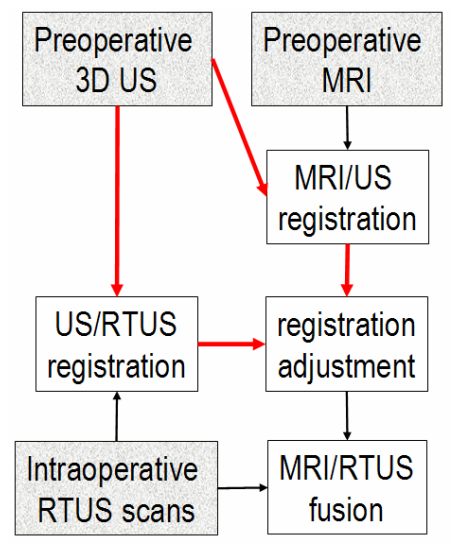

Fig. 2. Closed-loop control using feedback from real-time ultrasound (RTUS)

\section{Methods}

A closed-loop control system is proposed to account for prostate motion. The system uses intraoperative feedback to adjust the initial static registration between the MR and US images. The primary components of the system are shown in Figure 1. 


\subsection{Clinical Workflow}

The prostate MRI image is acquired first and transferred to a workstation. An endorectal coil is used to improve the MRI image quality and simulate the force of the ultrasound probe through the rectal wall although the degree of deformation is not exact. The MRI image can be obtained at any time before the biopsy. The patient is then positioned on an examination table and the 2D TRUS probe with tracking sensors is placed in the rectum. At the beginning of the TRUS procedure, the operator performs a 2D axial sweep (prostate base to apex) such that the series of $2 \mathrm{D}$ ultrasound images covers the entire volume of the prostate. The images and corresponding tracking data from the tracking sensors are transferred to the workstation in real-time. Based on these images and tracking data, a preoperative ultrasound volume is immediately reconstructed on the workstation [8]. Since all the 2D ultrasound images are tracked, the position and orientation of the reconstructed ultrasound volume can be determined in the tracking space. The MR image and ultrasound volume are then spatially aligned by manually adjusting a rigid-body transformation [7]. During the needle insertion and specimen acquisition, the operator manually holds the $2 \mathrm{D}$ probe to scan the prostate. Spatial tracking of the ultrasound probe, together with registering MRI image with the tracking coordinate system, enables real-time fusion of the live ultrasound image with the spatially corresponding multi-planar reconstruction (MPR) from the MRI scan. When prostate motion results in misalignment between the US and MR images, image-based registration between the real-time $2 \mathrm{D}$ ultrasound images and the preoperative ultrasound volume is carried out. The registration result is used to recover the correct MRI/US fusion image in the presence of prostate motion.

\subsection{Closed-Loop Control}

The closed-loop control is achieved by registering the real-time ultrasound images (RTUS) to the preoperative ultrasound volume as shown in Figure 2. The red arrows represent the closed loop. After the preoperative ultrasound volume is reconstructed, its position is fixed relative to the localizer, and can be used as a reference for motion compensation. As described in equation 1, the system uses feedback from the realtime scans to adjust the initial registration between the MRI image and the preoperative ultrasound volume, allowing for motion compensation of the prostate.

$$
T_{M R I \rightarrow R T U S}=T_{\operatorname{Pr} \text { op } U S \rightarrow R T U S} \bullet T_{M R I \rightarrow \operatorname{Pr} \text { op } U S}
$$

where $T_{M R I \rightarrow \operatorname{Prop} U S}$ is the initial manual registration between the preoperative US and MRI images carried out before the biopsy procedure; and $T_{\operatorname{Pr} \text { op US } \rightarrow R T U S}$ is a transformation determined by the online image registration between the real-time ultrasound images and the preoperative ultrasound volume.

It seems that the localizer plays no role in equation 1, meaning that in theory the closed-loop control can be achieved without the tracking system. However, the ultrasound transducer is held manually in any arbitrary position and orientation. The online image registration between the preoperative ultrasound volume and the real-time 
images can be extremely difficult if the spatial relationship between them is completely unknown. In addition, equation 2 requires the image-based registration to be conducted in real-time, which is very computationally expensive for current computers. The advantage of using the tracking system is that the registration only needs to be carried out when significant prostate motion occurs. In addition, the tracking system allows the transformation between the preoperative ultrasound volume and the RTUS imaging plane to be computed, thus providing a good starting point $\left(\hat{T}_{\operatorname{Pr} o p U S \rightarrow R T U S}\right.$ in equation 2) to initialize the image registration.

$$
\hat{T}_{\operatorname{Pr} \text { op US } \rightarrow \text { RTUS }}=T_{\text {Localizer } \rightarrow \text { RTUS plane }} \bullet T_{\operatorname{Pr} \text { op US } \rightarrow \text { Localizer }}
$$

\subsection{RTUS/US Registration}

It is initially assumed that the prostate is in the same location as it was during the $2 \mathrm{D}$ sweep, therefore the transformation between the current ultrasound image and the preoperative ultrasound volume can be estimated in equation 2 . The image-based registration takes the estimate as a starting point and performs numerical optimization. Since the starting point is determined by the tracking system and independent from the registration results of any other frames in history, it is always valid whether the processing of earlier frames succeeded or not. Given that the image registration may fail due to a lack of texture information, having an independent and robust starting point for each frame is critical.

The image registration algorithm is based on minimizing the sum-of-squared differences (SSD) between the current ultrasound image and the preoperative ultrasound volume. SSD is an attractive similarity measure for online registration because the mathematical formulation of SSD allows the objective function to be efficiently optimized using standard optimization algorithms such as the GaussNewton or the Levenberg-Marquardt algorithm.

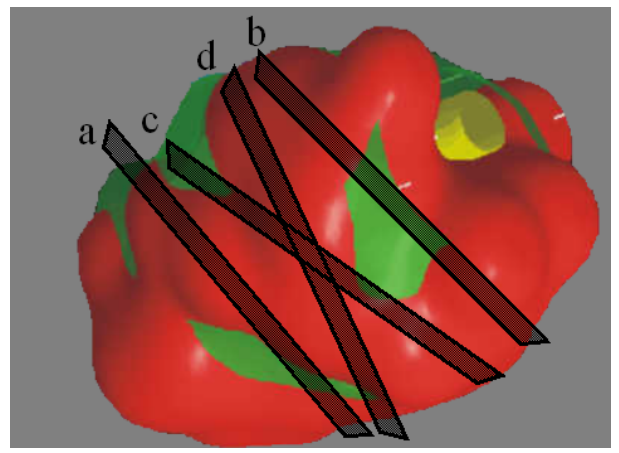

Fig. 3. Selected image frames for $2.5 \mathrm{D}$ to $3 \mathrm{D}$ registration

Since the spatial tracking of the ultrasound probe assigns a physical location in space to each image pixel, the $2 \mathrm{D}$ image is actually a single slice 3D image, allowing volume-to-volume registration to be conducted. However, the registration of the single slice volume is very sensitive to noise because there are many local minima along the off-plane direction, decreasing the algorithm's efficacy. It is therefore desirable to use more image frames for the registration. As an example illustrated in Figure 3, four ultrasound image frames are registered to the preoperative volume together. These four frames are selec-ted from a series of image frames in a short time period (e.g. 3 seconds). Since the probe is held manually, it is unlikely that the probe 
will be absolutely static. The motion of the operator can help to cover more prostate tissue in the off-plane direction. Using spatial tracking of the probe, two frames with the furthest translational distance are selected from the image series (Figure 3, a and b). The other two frames (Figure3, c and d) are selected due to their most different orientations. The registration between these frames and the ultrasound volume can be categorized as $2.5 \mathrm{D}$ to $3 \mathrm{D}$ registration. The objective function of the registration is

$$
O(\boldsymbol{\mu})=\sum_{k=1}^{N} \sum_{(x, y)}\left[I_{k}(x, y)-V\left(T_{k, \mu}(x, y, 0)\right)\right]^{2}
$$

where $N$ is the number of frame used in the registration, $I_{k}$ is the $\mathrm{k}^{\text {th }} 2 \mathrm{D}$ frame, $V$ is the preoperative ultrasound volume, $\mathrm{T}$ is a transformation model between $I_{k}$ and $\mathrm{V}$, and $\mu$ is a parameter vector. In our current implementation, a rigid-body transformation with six degrees of freedom (DOFs) is used to model prostate motion in the objective function. Other transformation models with higher DOFs (e.g. affine, quadratic etc...) may be able to account for some prostate deformation. However, the registration's robustness may be sacrificed. One interesting feature of our system is that the tracking error of the localizer (e.g. metal distortion) and the calibration error of the probe can be automatically corrected because of the direct image registration,

\section{Experiments and Results}

Both phantom and patient studies were carried out to evaluate the system. A 2D endocavity probe (C9-5, Philips Medical Systems, Andover, MA) was used to acquire 2D US images. The probe was tracked by attaching a disposable CIVCO (Kalona, IA) biopsy guide equipped with custom tracking sensors (Traxtal Inc., Toronto, Canada). The US images were captured using a frame-grabber card (Winnov, Sanata Clara, CA) and processed using custom software on a workstation with two $3.7 \mathrm{GHz}$ Dual Core Intel® Xeon ${ }^{\circledR}$ CPUs. The 2D sweep took 10 to 24 seconds. The reconstruction of the preoperative ultrasound volume took approximately 15 seconds using a speed enhanced algorithm and parallel computing [9]. The manual registrations between the MRI and ultrasound volumes were obtained in two to four minutes based on presegmented MRI images and some presets of prostate orientations [7]. The 2.5D/3D registration algorithm took about 15 seconds to compensate for prostate motion. The entire ultrasound procedure took approximately 15 minutes in patient studies.

\subsection{Phantom Study}

The system's accuracy was validated in a phantom study. A 6-DOF reference tracker was attached to a prostate biopsy phantom (CIRS, Norfolk, VA, USA). The global coordinate system was fixed on the phantom tracker and dynamic reference tracking [10] was used. Therefore, the prostate was static relative to the reference tracker. After the US volume was reconstructed, the $2.5 \mathrm{D} / 3 \mathrm{D}$ registrations were carried out to measure the prostate's position. An artificial error of 5 to $15 \mathrm{~mm}$ was introduced to the starting point of the registration. The error was uniformly distributed in 3D space. As an example shown in Figure 4, the registration starting point (Figure 4.c) was significantly different from the intraoperative image (Figure 4.a). Since the prostate 
was static in the reference coordinate system, a correct registration should recover the initial position. Figure 4.b shows the corresponding image of Figure 4.a in the US volume after the registration. The registration error of each voxel was defined as the distance from the recovered position to its original position. A total of twenty measurements were taken in the experiment, resulting in an error of $2.3 \pm 0.9 \mathrm{~mm}$.
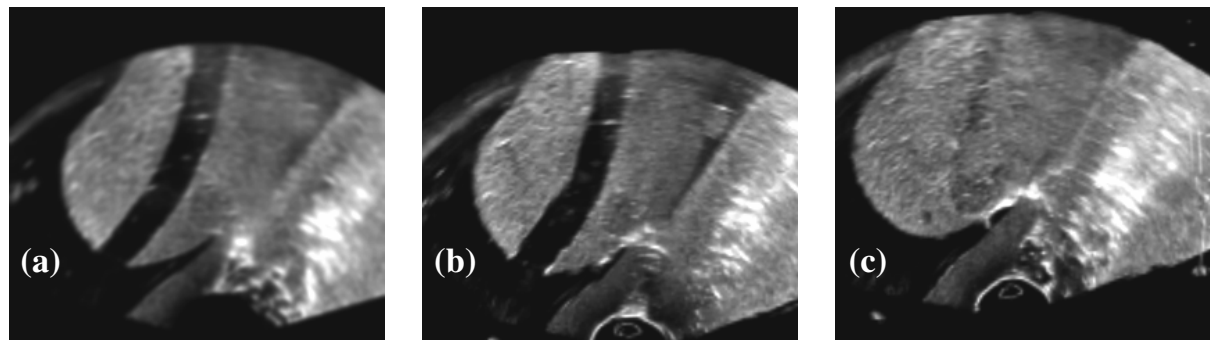

Fig. 4. Example of 2.5D/3D registration. (a) real-time ultrasound; (b) registration result of (a) in the reconstructed preoperative ultrasound volume; (c) initial starting point of the registration.

\subsection{Patient Studies}

The system was evaluated in patient studies from three perspectives. The capture range of the $2.5 \mathrm{D} / 3 \mathrm{D}$ registration was first tested. Figure 5 shows the objective function near the global minimum with respect to two translation parameters, giving an indication of the smoothness of the objective function and the likely capture range.

(a)

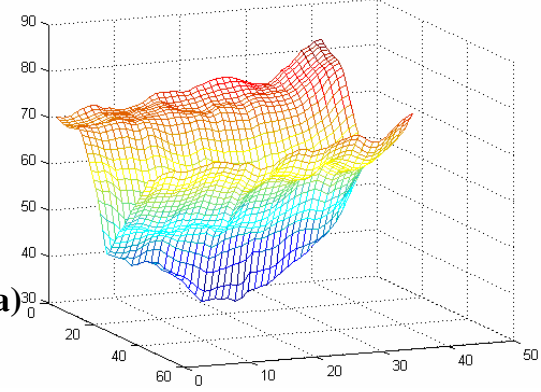

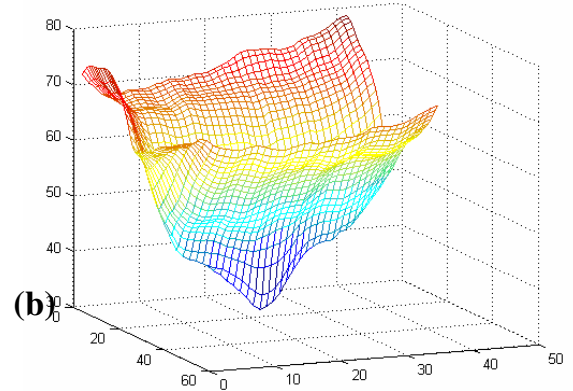

Fig. 5. Two-dimensional plots of the objective function near the global minimum with respect to two translation parameters. (a) is the result of registering one image frame. (b) is the result of registering four image frames. The grid unit is $1 \mathrm{~mm}$.

Figure 5 ( $\mathrm{a}$ and $\mathrm{b}$ ) are the results of registering one frame and four frames respectively. It can be observed that using more image frames results in a smoother objective function. The numerical optimization is therefore less likely to be trapped by local minima, making the registration more robust.

With the 2.5D/3D registration, the closed-loop control system was able to prospectively compensate for prostate motion in patient studies. As an example shown in 
Figure 6, the MRI volume is transformed to the 2D US image space. The red contours are the intersections of the prostate surface with the real-time US image. The segmentation was based on the MRI image and obtained before the biopsy procedure. After significant prostate motion was observed (Figure 6.b) in the image fusion, the image-based motion compensation was executed. As shown in Figure 6.c, the motion between the US and MRI images was well compensated.
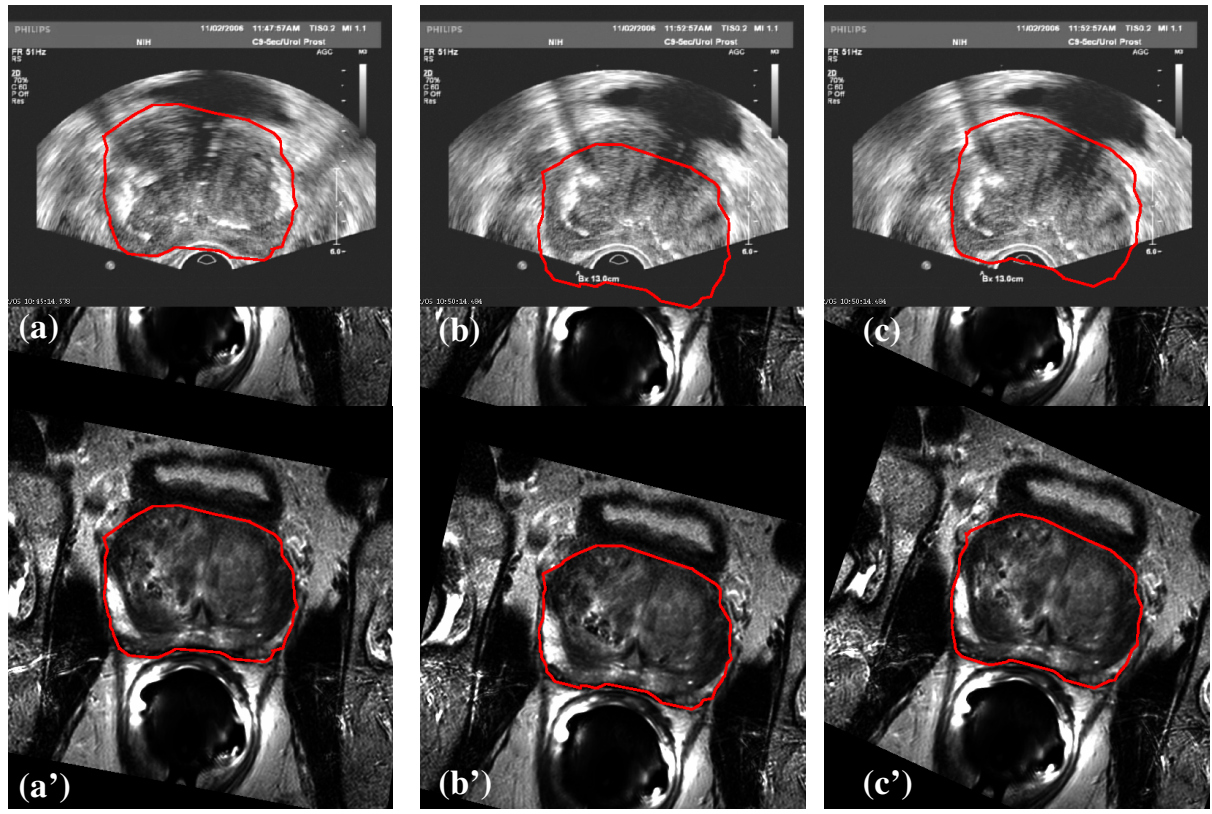

Fig. 6. Motion compensation using $2.5 \mathrm{D} / 3 \mathrm{D}$ registration. The red contours show the prostate segmentation in MRI image. The 3D MRI volume is pre-registered to a 3D ultrasound volume that is not shown. Top row: RTUS overlaid on MRI. Bottom row: MRI images. (a) and (a') are the initial registration without patient motion; (b) and (b') are the deteriorated registration after patient motion; and (c) and (c') are the registration after motion compensation.

The ultrasound image series and probe motion in patient studies were also recorded for retrospective analysis. A total of twelve patient studies were analyzed. At the time point of the motion compensation, one ultrasound image and two MR images (one each before and after the motion compensation) were saved for each patient. The prostate was then segmented from these $2 \mathrm{D}$ images by a radiologist and a radiation oncologist. The prostate segmentations of the MR images before and after the motion compensation were compared to the corresponding ultrasound segmentation respectively. The overlapping area of the MR and ultrasound segmentations was calculated. The results were normalized with the prostate's area of the ultrasound image. The analysis shows that the overlapping of the prostate between the MR and ultrasound images was $75 \% \pm 19 \%$ before the motion compensation and $94 \% \pm 5 \%$ after the motion compensation. The difference is statistically significant based on the students-t test $(\mathrm{p}<0.01)$. 


\section{Discussion and Conclusions}

This paper has presented a motion compensation system for prostate biopsy procedures using closed-loop control. The system takes advantages of both ultrasound and MRI imaging. Real-time fusion of MRI and ultrasound images can be obtained in presence of prostate motion. The tracking error of the localizer can be automatically accounted for using the image-based registration. Since only preoperative MRI images and 2D ultrasound scans are used, the system does not tie up MRI machine time for interventional procedures, providing a lower cost solution for MR guided prostate biopsy procedures. Patient studies show that the system is promising for clinical use.

The registration between the preoperative MRI and US images is currently done manually because it is the most reliable approach and the registration time seems to be clinically acceptable. It has been noticed that the physician can use the time needed for registration to examine the patient. However, robust and automatic MRI/US registration should be explored, since it is almost impossible to account for prostate deformation manually. The limitation of the current system is that the $2.5 \mathrm{D} / 3 \mathrm{D}$ registration time is not negligible. Therefore, the system is more effective for correcting large bulk motion than continuous motion.

\section{References}

1. American Cancer Society: Prostate Cancer, Statistics for 2007 (2007)

2. Fichtinger, G., Krieger, A., Susil, R.C., Tanacs, A., Whitcomb, L.L., Atalar, E.: Transrectal prostate biopsy inside closed MRI scanner with remote actuation, under realtime image guidance. In: Dohi, T., Kikinis, R. (eds.) MICCAI 2002. LNCS, vol. 2489, pp. 91-98. Springer, Heidelberg (2002)

3. Kaplan, I., Oldenburg, N.E., Meskell, P., Blake, M., Church, P., Holupka, E.J.: Real time MRI-ultrasound image guided stereotactic prostate biopsy. Magn. Reson Imaging 20(3), 295-299 (2002)

4. Schlaier, J.R., Warnat, J., Dorenbeck, U., Proescholdt, M., Schebesch, K.M., Brawanski, A.: Image fusion of MR images and real-time ultrasonography: evaluation of fusion accuracy combining two commercial instruments, a neuronavigation system and a ultrasound system. Acta Neurochir (Wien) 146(3), 276-277 (2004)

5. Krücker, J., Xu, S., Viswanathan, A., Shen, E., Glossop, N., Wood, B.J.: Clinical evaluation of electromagnetic tracking for biopsy and radiofrequency ablation guidance. Int. J. CARS 1, 169-171 (2006)

6. Malone, S., Crook, J.M., Kendal, W.S., et al.: Respiratory-induced prostate motion: Quantification and characterization. Int. J. Radiat. Oncol. Biol. Phys 48, 105-109 (2000)

7. Kruecker, J., Xu, S., Glossop, N., Guion, P., Choyke, P., Singh, A., Wood, J.B.: Fusion of real-time trans-rectal ultrasound with pre-acquired MRI for multi-modality prostate imaging. SPIE Medical Imaging 2007 (2007)

8. Trobaugh, J.W., Trobaugh, D., Richard, W.D.: Three dimensional imaging with stereotactic ultrasonography. Comput. Med. Imaging. Graph 18(5), 315-323 (1994)

9. Xu, S., Kruecker, J., Glossop, N., Wood, B.J.: Speed Enhanced Construction of 3D FreeHand Ultrasound. Computer Assisted Radiology and Surgery. Berlin, Germany (2007)

10. Glossop, N., Hu, R., Dix, G., Behairy, Y.: Registration methods for percutaneous image guided spine surgery. Computer Assisted Radiology and Surgery (1999) 